\title{
Randomness Representation in Turbulent Flows with Kolmogorov Complexity (In Mixing Layer)*
}

\author{
Masashi ICHIMIYA** and Ikuo NAKAMURA*** \\ **Institute of Technology and Science, The University of Tokushima, \\ 2-1 Minami-Josanjima-cho, Tokushima-shi, Tokushima, 770-8506, Japan \\ E-mail: ichimiya@tokushima-u.ac.jp \\ ${ }^{* * \star E}$ meritus Professor, Nagoya University
}

\begin{abstract}
Investigations of the definition and randomness of turbulence were reviewed. The Kolmogorov complexity measure of randomness was then introduced. Numerical and graphic data in the mixing layer formed downstream of a two-dimensional nozzle exit were compressed with the aid of a compression program. Approximated Kolmogorov complexity, AK, and normalized compression distance, NCD, were obtained. The AK indicated the regularity of the laminar flow and the randomness of the turbulent flow quantitatively. The NCD of the numerical value varied with data length. Between the same data, it approached zero, yet, between different data, it approached unity as the data length increased. The NCD of the numerical value in the natural transition process in the mixing layer increased monotonically downstream. Thus, the NCD appears to be the measure of the transition process. In the natural transition process in the mixing layer, the $\mathrm{AK}$ of the numerical value and the NCD of the graphic data did not change monotonously in the downstream direction. They therefore contain some uncertainty for measurement of the transition process.
\end{abstract}

Key words: Turbulence, Randomness, Kolmogorov Complexity, Normalized Compression Distance, Mixing Layer

\section{Introduction}

\subsection{Overview of Distinction Between Laminar and Turbulent Flows}

For irregularities from the transition process to developed turbulence of the mixing layer, one of the authors ${ }^{(1)}$ pointed out the effectiveness and disadvantage of the randomness factor $^{(2)}$ based on the distribution of the power spectrum of the turbulence. The present study follows a previous study and examines the problem in the irregularities from the viewpoint of "Kolmogorov complexity"(3) about an object, which recently has been studied in various fields.

Differences of laminar and turbulent flows are related to many fields in fluid engineering, e.g., pressure drop in a pipe, drag, heat transfer, flow induced vibration, noise, etc. From a scientific point of view, the main problems are why the difference in the laminar and turbulent flows appears, how to distinguish them ${ }^{(4)(5)}$ and the details of the flow in the turbulence. There is a strong correlation between the two fields, of course, and from the scientific point of view, details of the turbulence have been examined. The basis of the current numerical simulation of turbulence was then established and developed. Conventionally, the turbulent flow has been exclusively regarded as important in 
engineering fields. However, since the following facts became known, there is an increasing importance of transition studies. That is, the Reynolds number in microfluidic devices can span both laminar and turbulent regime, and an intermittent transition phenomenon in circular pipes can be seen in the transition of blood flow, which may relate to damage of the vessel wall ${ }^{(6)}$.

For a long time, there has been interest in various phenomena in fluid science, e.g., visualization and visual output of hot-wires at the instantaneous clear boundary between the turbulent boundary layer and outer laminar region, wall- and free-shear turbulent flows exhibiting "coherent structure"(4) in random environments; and its strong relationship with the Reynolds shear stress is recognized in wall turbulence, turbulent puffs or slugs appearing in transitional circular pipes and turbulent spots in the transitional boundary layer and their boundaries are clear but irregular in time and space ${ }^{(5)(7) \sim(9)}$. This paper concerns identification of the above nature of the turbulence.

\subsection{Definition of Turbulence}

Since the distinction criterion between turbulent and laminar, i.e., non-turbulent, flows is a key issue in this kind of research, the history of the definitions of turbulent and laminar flows is reviewed here. Reynolds stated that "the motion of water shall be direct or sinuous" and did not use the term "laminar" or "turbulent." ${ }^{(10)}$ Prandtl cited the experiment of Reynolds and showed that "smooth flow is laminar, on the other hand, vortical flow is a turbulent flow." ${ }^{(11)}$ Similar definitions have been used in many textbooks on fluid mechanics. Tani's definition, based on subsequent knowledge of the nature of turbulence, is "laminar flow is the condition in which fluid flows orderly and laminarly, on the other hand, turbulent flow is the condition in which fluid flows irregularly mixed." ${ }^{(12)}$ Recently, Schlichting and Gersten used almost the same definition ${ }^{(13)}$. Theoretical books do not use phrases such as "the turbulence is condition whose property is ...," but start from the distinction between mean and irregularly fluctuating velocities ${ }^{(14)(15)}$.

It may be Hinze who first gave a detailed definition of turbulence ${ }^{(16)}$. He supplemented the definitions of Taylor and von Karman and expressed "turbulent fluid motion is an irregular condition of flow in which the various quantities show a random variation with time and space coordinate, so that statistically distinct average values can be discerned" and gave the addition of "with time and space coordinate." On the other hand, Townsend ${ }^{(17)}$, and Tennekes and Lumley ${ }^{(18)}$ did not give a definition similar to that of Hinze, but indirectly defined turbulence by enumerating its nature. Tennekes and Lumley ${ }^{(18)}$ described it as "irregularity, diffusivity, large Reynolds numbers, three-dimensional vorticity fluctuations, dissipation, continuum and turbulent flows are flows." One of the authors follows their definition $^{(19)}$. All of the examples above noted that turbulence is irregular and a random phenomenon, but did not to explain what is random.

In the practical treatment of turbulence based on values of turbulence intensity or the time derivative of velocity fluctuations, the definition function $I(x, t)$ for turbulent flow is usually introduced $^{(7)(20)}$. The definition function is set unity or zero in the case of turbulence or non-turbulence, respectively. The idea is the same as the defining function or the characteristic function in probability theory and various fields, such as multiphase flows, although some subjectivity is introduced in the distinction.

The discovery of chaos, especially random numerical solutions in the ternary system of deterministic nonlinear ordinal differential equations stemming by Lorenz ${ }^{(21)}$ from investigations of thermal convection, had great influence on not only turbulence but also randomness in many natural phenomena. As a result, the study of fluid motion based on the dynamical systems founded by Poincare can be said to have begun. Before then, only mathematicians were interested in dynamical systems ${ }^{(22)}$. Recently, Pope confronted irregular or random flows, although his main definition remained as before ${ }^{(23)}$. 


\section{Randomness}

\subsection{Concept of Randomness}

For the definition of the turbulence covered in the previous section, an essential property is expressed verbally in terms of irregular, random or chance. Since the goal of the present study is to examine the degree of turbulence in relation to randomness rather than the intensity of fluctuating vorticity or value of the Kolmogorov scale, first we state what randomness means and how to measure it.

Situations closely related situations to the measurement of randomness are probability and likelihood. Laplace is very helpful on this issue ${ }^{(24)}$. Following him, a prominent contributer about randomness is Poincare ${ }^{(25)}$, who is the founder of the theory of dynamical systems. Pope defined randomness in relation to turbulence ${ }^{(23)}$. His definition seems reasonable and practical, however, his definition cannot be used to measure randomness, and relating it to Kolmogorov's probability axiom is difficult. Kolmogorov himself described the application of probability theory, but did not mention random itself at that time ${ }^{(26)}$.

\subsection{Fundamental Random Subjects}

Fundamental subjects to study randomness mathematically include discrete binary finite or infinite strings, e.g., $\{0,1\}^{6}:=\{0,1,1,1,0,0\}$ or $\{0,1\}^{*}:=\{1,1,0,1,0, \ldots\}$ and finite or infinite curves e.g., results of finite-time observation of a particle moving with Brownian motion and results of infinite time tracking of the evolution of fluid particles in turbulent flow from the Lagrangian viewpoint by G. I. Taylor. Historically viewed in terms of mathematical development ${ }^{(27)(28)}$, von Mises began the study of infinite discrete randomness in 1919, and Martin-Löf gave a complete definition in the discrete infinite case in $1966^{(29) \sim(31)}$.

The degree of randomness of finite strings is currently discussed with the aid of Kolmogorov complexity, $K(x)$, which will be described in the next section $^{(27)(28)(30)(31)}$. Data from hot-wire anemometers described in this paper are finite or ideally infinite time curves. On the other hand, a representative of the analytical random curves is a mathematical theory of Brownian motion that has been treated widely with the development of the theory of stochastic differential equations of Ito or appears in fluid flows as an expression of random terms in some equations, such as fluid diffusion ${ }^{(23)(32)(33)}$ or representation of turbulent velocity $^{(34)}$. However, theories that discuss randomness of experimental curves in turbulent data directly are missing within the authors' knowledge. Moreover, because in the real turbulent experiments the data are discrete strings digitized within finite time, the data can be the subject of the Kolmogorov complexity of finite strings.

Reports discussing the complexity of the phenomenon from the perspectives of dynamics or physics include the following within the authors' knowledge. Gurzadyan ${ }^{(35)}$ proposed the Kolmogorov complexity of anisotropy spots of cosmic microwave background radiation sky maps as a new descriptor for revealing crucial cosmological information, though the validity is unclear since he did not discuss the actual data. Gell-Mann and Lloyd discussed the problem of complexity from the coarse-grained viewpoint of statistical mechanics ${ }^{(36)(37)}$. Takeda discussed Gell-Mann's idea in relation to the human brain ${ }^{(38)}$. Kreinovich and Kunin discussed the relation of chaos and complexity ${ }^{(39)}$ and suggested the use of the ideas of the Kolmogorov complexity to distinguish singular and non-singular solutions. Badii and Politi discussed instability and turbulence of flows at the beginning of complex examples ${ }^{(40)}$. There is also the concept of stochastic complexity of Rissanen ${ }^{(41)}$, though the problem is beyond the scope of this study. 


\section{Kolmogorov Complexity and Compressibility}

In this section, we briefly describe Kolmogorov complexity and normalized compression distance ${ }^{(27)(28)(30)(31)}$ to facilitate understanding of the contents of this paper. Kolmogorov related computational and information theories to deal with the randomness of finite sequences with computers defined by the Kolmogorov complexity, $K(x)$, of discrete finite strings, $x$, belonging to a set, $D$, by Eq. (1). The strings are thought to be binary coded and are shown in lower case in accordance with the custom in this field. As a computer and programming language, some system, $S$, is selected and specified.

$$
K(x)=\min \{|P|: S(P)=x\} .
$$

Where $P$ means a certain program written in the specified language, $|P|$ means its length, $|x|$ means the length of the object, $x$, and the equation $S(P)=x$ means that $x$ is calculated when the program $P$ is run on the system, $S$. That is, $K(x)$ is the length of the shortest program for outputting the object $x$. If there is some regularity in the object, the program to calculate the object can be shortened if one reflects the regularity in the programming. However, if there is no regularity, that is, if the object is genuinely random, there is no way to show the object by the computer by creating a program that shows the object as it is.

The randomness of the object, $x$, is defined by $k$-randomness in the following equation for some constant, $k^{(31)}$.

The object, $x$, is $k$-randomness $\Leftrightarrow K(x) \geq|x|-k$.

If $k$ is small, the randomness is strong, and in this situation $k$ can be set as zero. At this time, if the object, $x$, is random, the minimum value of $K(x)$ is larger than or equal to $|x|$. That is, if $x$ is random at the level of $k=0$, only one program, $P$, written to express the object itself exists for the output. Therefore, the length of this $P$ is naturally longer than $|x|$. In this way, the measurement of the randomness of the object, $x$, becomes possible with $K(x)$. It also has been demonstrated that there is a close relationship between Martin-Löf randomness of the infinite strings mentioned above and $K(x)^{(31)}$. This property presents a way to compress the length of the object, $x$. This condition was expressed by Vitányi ${ }^{(42)}$. Roughly speaking, for a given string, $x$, the Kolmogorov complexity, $K(x)$, was the bit length of the binary strings obtained when compressed ultimately within a range where it could decoded. For a discussion on understanding of the flow and Kolmogorov complexity, see Ref. (43).

\section{Data Compression for Approximated Kolmogorov Complexity}

\subsection{Relative Kolmogorov Complexity and Data Compression}

Kolmogorov proved various properties about $K(x)$, especially the above-mentioned invariant theorem, then showed its effectiveness for the study of randomness of finite strings. However, it has proven impossible to calculate $K(x)$ as well as the halting problem of the Turing machine ${ }^{(31)}$. Therefore, it is unlikely to utilize $K(x)$ for actual data, though it is an effective quantity for the pure theory of the randomness. To overcome this shortcoming, as a way to approximate $K(x)$, a method to utilize a practical compression program has been proposed from the viewpoint of the previous section ${ }^{(42)(44)}$. That is, as an approximation of $K(x)$, to use a bit length, $C(x)$, of the compressed data, $x$, obtained by the practical compression programs.

In this paper, to examine experimental data, i.e., digitized output of hot-wire anemometers, and approximate their complexity, we used the ratio of the length of the compressed data length by the program, $C$, and the original data length, 
$\mathrm{AK}(x)=C(x) /|x|$

and designated the approximated Kolmogorov complexity, AK. This is a simple approximation of complexity, although it depends on the performance and property of the compression program, of course. The performance of the compression program is discussed in Section 5.2.

In practice, to distinguish the complexity between elements belonging to different sets is more important than to consider a single complexity itself. The fundamental aspect of the distinction is the distance defined in the set. Kolmogorov defined the relative Kolmogorov complexity for two objects, $x$ and $y$, in the following equation.

$$
K(x \mid y)=\min \{|P|: S(P, y)=x\} .
$$

This is the shortest length of the programs to calculate $x$ with supplementary information, $y$. In other words, $K(x \mid y)$ is the length of the shortest program to compute $x$ when one enters the auxiliary information $y$ in the computer. The information $y$ can be said to be a hint of examination $x$. For the compression, it is the ultimate length of the compressed string when it is decoded with the auxiliary data, $y^{(42)}$. With $y$ to compress $x, K(x \mid y)$ may be much smaller than $K(x)$. The difference between them can be considered as an improvement of the compression efficiency in the case when one compresses $x$ under the assumption of $y$, rather than without any knowledge of $y$. Therefore, the difference $K(x)-K(x \mid y)$ can be estimated as the amount of information of $x$ contained in $y$. In this regard, the difference, $K(x)-K(x \mid y)$, has been shown to be the maximum information amount of $x$ in $y$, and the difference can be approximated as $K(y)-K(y \mid x)$. Therefore, the difference can be regarded as the amount of similar parts between $x$ and $y^{(3)(42)}$.

With the relative Kolmogorov complexity, the information distance between the data, is defined as, $E(x, y)=\max \{K(x \mid y), K(y \mid x)\}$. The information distance is shown to satisfy the distance axioms, i.e.,

$$
\left.\begin{array}{l}
d(x, y) \geq 0, \\
d(x, x)=0, \\
d(x, y)=d(y, x), \\
d(x, y)+d(y, z) \geq d(x, z) .
\end{array}\right\}
$$

That is, non-negativeness, identity, symmetry and triangle inequality except for additive constants. Based on the information distance, the normalized information distance (NID) represented by the following equation is considered and its theoretical properties have been investigated $^{(44)}$.

$$
\mathrm{NID}(x, y)=\frac{\max \{K(x \mid y), K(y \mid x)\}}{\max \{K(x), K(y)\}} .
$$

Note that Eq. (6) corresponds to Eq. (9) in the next section.

\subsection{Normalized Compression Distance Between Objects with Data Compression}

Though NID is appropriate as a quantity that determines the differences between different data theoretically, it also cannot be calculated as $K(x)^{(44)}$. Therefore, a corresponding use of $C(x)$ for an approximation of $K(x)$ has been proposed as the normalized compression distance (NCD). First, the relative complexity in the compression is defined with the quantity $C(x \mid y)=C(y x)-C(y)$. The data $y x$ can be made by simply connecting the data $x$ after the data $y$, then the quantity $C(y x)$ is the length of the compressed data, $y x$. Intuitively, it is considered reasonable for the compression of $y x$ to 
first compress $y, C(y)$, then to compress $x$ with the aid of the result, $C(x \mid y)$. Hence, the above quantity is considered reasonable. Therefore, these expressions can be used to approximate the Kolmogorov complexity ${ }^{(42)(44)}$. Then, the following equation holds:

$$
C(x)-C(x \mid y)=C(x)+C(y)-C(y x) .
$$

This is the approximated value of the similarity between $x$ and $y$. As a normalized similarity, $\{C(x)+C(y)-C(y x)\} / C(x)$ can be considered. However, since a measure of the distinction or dissimilarity, i.e., distance, is required, the similarity which is subtracted from unity is used below as the distance,

$$
1-\frac{C(x)+C(y)-C(y x)}{C(x)}=\frac{C(y x)-C(y)}{C(x)} .
$$

The normalized compression distance, NCD, between object $x$ and $y$ is defined by the following equation.

$$
\operatorname{NCD}(x, y)=\frac{\max \{C(x y)-C(x), C(y x)-C(y)\}}{\max \{C(x), C(y)\}} .
$$

Assuming that $C(x y)=C(y x), \mathrm{NCD}$ is expressed by the following expression.

$$
\operatorname{NCD}(x, y)=\frac{C(x y)-\min \{C(x), C(y)\}}{\max \{C(x), C(y)\}} .
$$

This is used as the defining equation of NCD. By utilizing NCD, clustering of practical data becomes possible. The expressions are applied to a classification of languages and novels $^{(42)}$, animals ${ }^{(44)}$, image searches ${ }^{(45)}$, evaluation of valuation of services ${ }^{(46)}$, and words in search engines on the $w_{e b}{ }^{(47)}$.

\section{How to Determine Specific NCD in This Study}

\subsection{Compression Work}

In this section an actual method for determining NCD will be explained. Until Section 4 , the data are expressed in lower-case letters as $x$ or $y$ according to many references, but in this and subsequent sections, lower-case letters mean experimental coordinates in Fig. 4, and the data are expressed in capital letters, $X$ or $Y$. Two types of data are used for the compression: text files of numerical data, and bitmap files of image data. In the case of numerical data, $X Y$ is made by simply connecting data $Y$ after data $X$. Therefore, the file size of data $X Y$ is the sum of that of the data $X$ and $Y$. Further, in the image data, $X Y$ is made in drawing software; the image of the data $Y$ is to be located without space on the upper side of the image data of $X$. Since the format of the bitmap file is, after the 54-byte header section for expressing various information, filling an image bitmap of three bytes per pixel. In this case, due to the existence of the header section, the file size of the data $X Y$ is not strictly equal to the sum of that of the data $X$ and $Y$. In compression schemes, files are compressed using a compression program running on a Windows PC. At this time, by substituting file sizes of the uncompressed $|X|$ and calculated $C(X), C(Y)$ and $C(X Y)$ to Eqs. (3) or (10), we obtain $\operatorname{AK}(X)$ and $\operatorname{NCD}(X, Y)$.

\subsection{Decision of Compression Format}

The optimal compression format for NCD is decided among various formats. Six widely used types, 7z, RAR, bzip2, LZH, ZIP, gzip, are tested. Both the numerical and 
image data described in the previous section were tested. First, for the numerical data, the streamwise fluctuating velocity, $u$, was measured in the laminar-turbulent transition process of the mixing layer described in Section 6 by the hot-wire anemometer, then digitized at intervals of $0.2 \mathrm{~ms}$. From each data set the minimum value was subtracted, then normalized by a constant common to all measurement data not to exceed unity, and converted to a 3 -digit integer. Therefore, one velocity datum corresponds to an integer in the range of 000-999. Integer values less than 99 are aligned with the 3-digit integers by adding one or two 0 s to their beginning. Since the 3 -digit integer is proportional to the original velocity, the range is wider in turbulent flow than in laminar flow. The reason for this conversion is to remove the decimal point and negative sign from the original data. Further, since the output of the hot-wire anemometer is A/D converted in 12 bits, i.e., 4096 steps, in order to match the order, the converted data are divided in 1000 steps. Such 98304 integer numbers (32768 velocity data) aligned in a row make a text file whose capacity of 98304 bytes. For example, in a laminar flow position described later, at $x / h=0.5, y /(h / 2)=0$, the first nine of the 98304 integers (three velocity data) are "005005005." In a turbulent flow also described later, at $x / h=8, y /(h / 2)=1.3$, they are "251257269." The image data form a 480054-byte bitmap file of each 400 pixels in width and height, where 262144 rectangles ( 2 pixels on the long side and 1 pixel on the short side) were scattered in the range from -1 to 1 in $u / U_{0}$ $v / U_{0}$ plane (e.g., Fig. 13), described later in Section 6.5 in detail.

The compression format is decided from two points of view. First, to satisfy the identity axiom, $\operatorname{NCD}(X, X)=0$ in Eq. (5) as far as possible. For the data $X$, as the representative position in the laminar and turbulent flows, on the center line just behind the nozzle, $x / h=$ $0.5, y /(h / 2)=0$, and the maximum position of rms value of the streamwise fluctuating velocity, $u$, within the range of $x / h \leqq 20, x / h=8, y /(h / 2)=1.3$, are chosen, respectively. The coordinates and symbols are shown in Fig. 5.

Table 1 shows NCD between the same data. Among all formats, NCD in $7 \mathrm{z}$ format is closest to zero. That is, the identity axiom is most satisfied with $7 \mathrm{z}$. On the other hand, in LZH, ZIP and gzip, NCD is larger and about unity. In $7 \mathrm{z}$ format, the numerator of $\mathrm{NCD}(X$, $X)(=C(X X)-\min \{C(X), C(X)\}=C(X X)-C(X))$ was approximately zero, since the capacities of $C(X)$ and $C(X X)$ were almost the same. In LZH, ZIP and gzip formats, the numerator was approximately equal to the denominator ( $\max \{C(X), C(X)\}=C(X))$, since $C(X X)$ became about twice $C(X)$. That is, in the $7 \mathrm{z}$ format, the result of the compression of the combined data $X X$ may be coded as, e.g., "twice THE COMPRESSION OF $X$ ", and the length "twice" is negligibly small compared with that of the single data $X$, "THE COMPRESSION OF $X^{\prime \prime}$, then $C(X) \simeq C(X X)$. In LZH, ZIP and gzip formats, the result of the encoding of $X X$ may be, e.g., "THE COMPRESSION OF $X$, THE COMPRESSION OF $X$," and the capacity is about twice the capacity of $X$. Though such differences may be due to their attributes, in the present study we avoid discussing compression performance and focus on finding the most suitable compression format for the calculation of NCD.

The second requirement the compression format needs to satisfy is the symmetry axiom, $\operatorname{NCD}(X, Y)=\operatorname{NCD}(Y, X)$ as far as possible. The datum $X$ was the representative position of laminar flow as above, $x / h=0.5$, the $y /(h / 2)=0$. For data $Y$, one is in the laminar flow and very close to the reference position, $x / h=0.5, y /(h / 2)=0.2$, the other is in the aforementioned turbulent position, $x / h=8, y /(h / 2)=1.3$. Table 2 shows the difference,

Table 1 NCD between same data

\begin{tabular}{|c|c|c|c|c|}
\hline \multirow{2}{*}{ Dormat } & \multicolumn{2}{|c|}{ Numerical } & \multicolumn{2}{c|}{ Graphic } \\
\cline { 2 - 5 } & Laminar & Turbulent & Laminar & Turbulent \\
\hline 7z & 0.013 & 0.003 & 0.315 & 0.062 \\
\hline RAR & 0.045 & 0.007 & 0.635 & 0.969 \\
\hline bzip2 & 0.355 & 0.381 & 0.053 & 0.288 \\
\hline LZH & 0.973 & 0.992 & 0.904 & 0.967 \\
\hline ZIP & 0.979 & 0.975 & 0.703 & 0.971 \\
\hline gzip & 0.969 & 0.957 & 0.906 & 0.990 \\
\hline
\end{tabular}

Table 2 Difference of $\operatorname{NCD}(X, Y)$ and $\operatorname{NCD}(Y, X)$

\begin{tabular}{|c|r|r|r|r|}
\hline \multirow{2}{*}{ Data $Y$} & \multicolumn{2}{|c|}{ Numerical } & \multicolumn{2}{c|}{ Graphic } \\
\cline { 2 - 5 } & Laminar & Turbulent & Laminar & Turbulent \\
\hline 7z & -0.003 & 0.005 & 0.019 & 0.001 \\
\hline RAR & 0.001 & -0.002 & 0.000 & 0.000 \\
\hline bzip2 & 0.000 & 0.000 & -0.011 & -0.003 \\
\hline LZH & 0.006 & 0.007 & 0.000 & -0.008 \\
\hline ZIP & 0.001 & -0.004 & 0.000 & 0.000 \\
\hline gzip & 0.001 & -0.001 & 0.000 & 0.000 \\
\hline
\end{tabular}


$\operatorname{NCD}(X, Y)-\mathrm{NCD}(Y, X)$, in the case where $Y$ is in the form contained in the first line in Table 2 . Values are very small in any compression format, therefore, the symmetry is regarded as satisfied. From the above, the optimal compression format for NCD is regarded as $7 \mathrm{z}$, therefore, we decided to use $7 \mathrm{z}$ for the subsequent analysis. For reference data $X$, data on the centerline just behind the nozzle, $x / h=0.5, y /(h / 2)=0$ are to be used in the subsequent analysis. The two remaining axioms were tested in $7 \mathrm{z}$ only, where a non-negative value was held for all data calculated, and triangle inequality was also established for many examples.

\subsection{Effect of the Number of Numerical Data}

In this section, an appropriate data length for the numerical data was determined. First, as in the previous section, to examine the identity axiom, NCD between same numerical data, $\operatorname{NCD}(X, X)$, was calculated. The data $X$ was the same form as used in section 5.2, using the $\mathrm{n}$ integers from the beginning of 786432 integer data with 12 kinds of $n$ changing from 384 to 786432, then twelve NCD's were calculated. As in Table 1, the laminar flow between the reference position data, and turbulent flow data at $x / h=8, y /(h / 2)=1.3$, were used. Figure 1 shows the variation of NCD as a function of the data length. Except between the laminar flow and $n<6 \times 10^{3}$, the larger the data length the nearer NCD approaches zero. Since the greater increase in the length of the "THE COMPRESSION RESULT OF $X$ " in the previous section, the relative length of the "twice" becomes negligibly smaller, and $C(X)$ tends to be closer to $C(X X)$. The reason for the greater smallness of NCD in the turbulent flow than the laminar flow is that randomness is large in the turbulent flow, and the length of the "THE COMPRESSION RESULT OF $X$ " is larger than in the laminar flow with the same data length. From Fig. 1, it can be seen that the data length is preferably as long as possible.

For the symmetry axiom, variation of $\operatorname{NCD}(X, Y)-\operatorname{NCD}(Y, X)$ with the data length is shown in Fig. 2. As well as Table 2, the position of the reference data, $X$, is on the centerline just behind the nozzle. The position of the data $Y$ is $x / h=0.5, y /(h / 2)=0.2$ as the representative of the laminar flow and the $u$ '-maximum position as the representative of the turbulent flow. The value fluctuates considerably with the data length as long as it is short, whereas it gradually approaches zero as the data length increases and the symmetry axiom obtains. From the results of Fig. 2, greater data length is desirable.

Next, in the actual calculation of NCD, the two data, $X$ and $Y$, are always different, and $\operatorname{NCD}(X, Y)$ as a function of $n$ is shown in Fig. 3. Laminar and turbulent flows in data $Y$ are the same as those in Fig. 2. The NCD will increase with increasing $n$ whether data for $Y$ is laminar or turbulent. For $n>2 \times 10^{4}$ in turbulent flow NCD becomes constant, approximately unity. Since an integral time scale of the fluctuating velocity is approximately $20 \mathrm{~ms}$ (= 100 sampled data) or $1 \mathrm{~ms}$ (= five sampled data) in laminar or turbulent flows, respectively, $n$ (= 3 times of sampled data) in Fig. 3 is a sufficiently long period where autocorrelation is already zero. Hence, as $n$ increases, the randomness may be strengthened, and NCD increases. In addition, NCD is greater in turbulent than laminar data for $Y$, since the difference of the randomness between the reference laminar data is greater. From the viewpoint of the distinction between laminar and turbulent with NCD, the

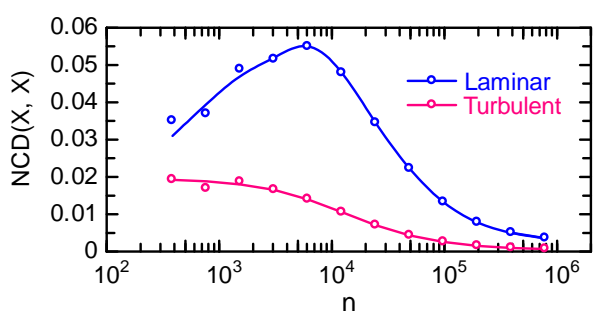

Fig. 1 Distribution of NCD between same data as a function of data length.

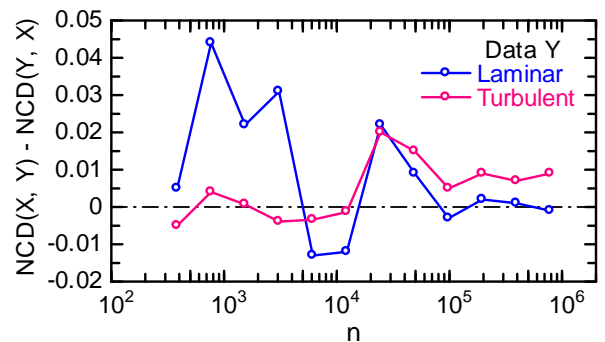

Fig. 2 Difference of $\operatorname{NCD}(X, Y)$ and $\operatorname{NCD}(Y, X)$ as a function of data length. 


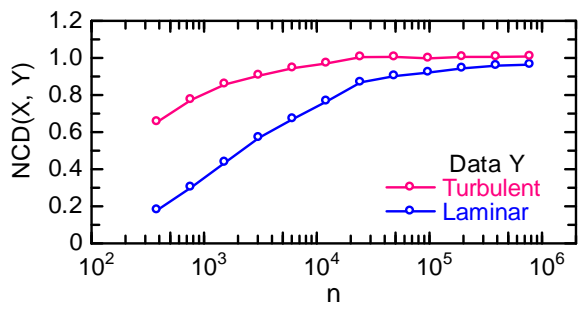

Fig. 3 Distribution of NCD between different data as a function of data length.

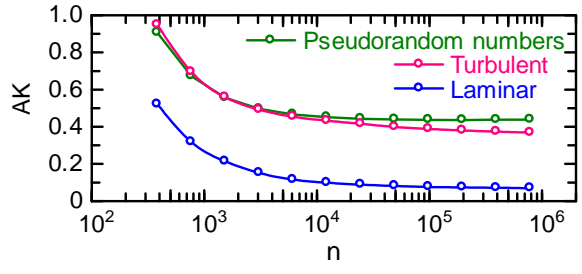

Fig. 4 Distribution of AK as a function of data length.

difference of NCD between laminar $Y$ and turbulent $Y$ is preferably larger, and $n$ is preferably smaller. However, the range $n<2 \times 10^{4}$, where $\mathrm{NCD}$ varies with $n$, is undesirable.

The change of AK with data length is shown in Fig. 4. Turbulence and laminar data are the same as Fig. 1. In addition, the pseudo-random number of the 3-digit integer of the same type as the velocity data is created based on Mersenne Twister ${ }^{(48)}$, which is regarded as good quality. In all data, AK decreases as $n$ increases, though the decrease gradually slows. If the random number is completely random, $C(X)$ equals $|X|$, and $\mathrm{AK}$ then becomes unity since the random number cannot be compressed. Therefore the fact that $\mathrm{AK}$ in the pseudo-random number decreases with the increase of $n$ indicates the pseudo-nature of the random number generation method. That is, since the random number is made by a program of finite length, with the increase of the denominator of the defining equation of AK, Eq. (3), AK may decrease. The reason for the gradual saturation may be that the numerator, $C(x)$, is not the original program length of the random string generation but the compressed data length. Since it is undesirable that AK varies widely with $n$, a greater $n$ is also desirable in Fig. 4 .

From the results in Figs. 1-4, we decided to use $n=98304$ (number of fluctuating velocity data $=32768$ ). For this reason, the capacity of all numerical data is 98304 bytes and that of the combined data $X Y$ is the sum of those of the single data, 196608 bytes. The number of fluctuating velocity data, 32768, corresponds to approximately 6.6 seconds in actual acquisition time of the $\mathrm{A} / \mathrm{D}$ conversion.

In addition, the effect of the time interval of the data on NCD and AK was also quickly checked. While keeping the total time of 6.6 seconds constant, the time interval the number of data varied. As the time interval varied, the value of AK and NCD varied. However, as the time interval became smaller, the result tended not to vary, and finally, at intervals of one order of magnitude smaller than the integral time scale the values became almost constant. Therefore, the time interval employed in this study, the same as the sampling interval of the A / D conversion, $0.2 \mathrm{~ms}$, is considered satisfactory. As can be seen from Fig. 3 , NCD between the reference data and the laminar-flow data exceeds 0.9 , while that between the reference data and the turbulent-flow data becomes approximately 1.0. The following discussion pertains to conditions where NCD varies within the range of 0.1 when $Y$ is between laminar and turbulent flows. For $Y$ in the case of using the same data as the reference position $X, \mathrm{NCD}$ is 0.013 as shown in Fig. 1 and Table 1 . When using the close proximity of $X$, at $x / h=0.5, y /(h / 2)=0.2$, NCD becomes 0.921 as shown in Fig. 3. Hence, even within the same laminar flow, between different data NCD will exceed 0.9.

\section{Results and Discussion}

\subsection{Mixing Layer for Analysis}

In this section, we describe the result of the complexity analysis of the data measured in the mixing layer. The mixing layer is formed at the beginning of the jet ejected into a quiescent fluid from a rectangular nozzle. We measured the distributions of the mean and 


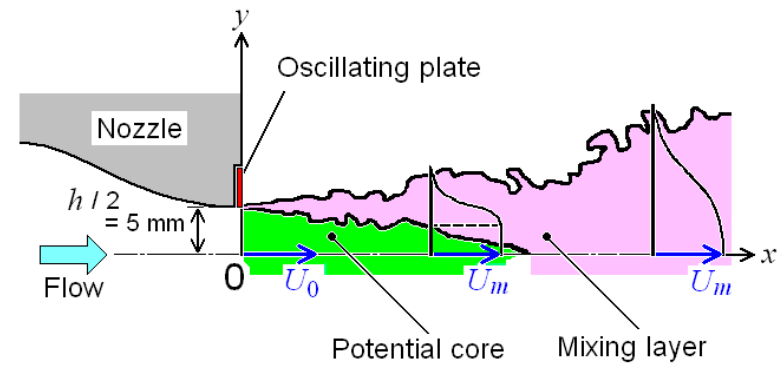

Fig. 5 Schematic diagram of two-dimensional mixing layer and coordinate system.

fluctuating velocities, spreading of the jet, production, convection and dissipation rates of the fluctuating energy ${ }^{(49)}$. We also revealed the progression of the laminar-turbulent transition process in the flow-normal direction, and obtained the randomness factor ${ }^{(1)}$ which was proposed by Sato ${ }^{(2)}$ as an indicator of the transition progress, then examined its validity in the transition process.

The experimental apparatus is the same as that shown in previous report ${ }^{(1)(49)}$. The jet is ejected to the quiescent fluid from the exit $(x=0)$ of a two-dimensional nozzle whose width of $310 \mathrm{~mm}$ and height, $h$, of $10 \mathrm{~mm}$. For the convenience of the reader, the coordinate system and the upper half of the flow field are reproduced in Fig. 5. In the previous report, two cases of disturbance were reported. In one case, two thin plates installed at the exit of the nozzle were stationary so as not to narrow the nozzle exit section, in the other case the plates oscillated perpendicular to the jet and symmetrically with respect to the centerline with a frequency of $5 \mathrm{~Hz}$ and a respective amplitude of $0.25 \mathrm{~mm}$. In this paper, only the case where the plates are at rest is reported. How complexity varies in the stationary and oscillating states will be reported in the future.

The experimental condition is also exactly the same as the previous report. The Reynolds number based on the height, $h$, and the nozzle exit velocity, $U_{0}$, was kept at 5000 $\left(U_{0} \simeq 7.5 \mathrm{~m} / \mathrm{s}\right)$. X-shaped hot-wire probes with two tungsten sensing elements, each $5 \mu \mathrm{m}$ in diameter and $1 \mathrm{~mm}$ in length, were used for the measurements. Output voltage was sampled at a frequency of $5 \mathrm{kHz}$ for about 52 seconds. The measurements were conducted in a range of $y \geqq 0$. Results are shown here in the range $x / h \leqq 20$, where self-preservation is well established.

\subsection{Conventional Measure for Laminar-Turbulent Transition of Mixing Layer}

As described in the previous section, Sato et al. proposed a randomness factor defined as the ratio of the energy of the continuous spectrum to the total energy in the power spectrum distribution of the velocity fluctuations ${ }^{(2)}$. In the previous report, the power spectrum and the probability density function of the velocity fluctuation were obtained, and the applicability of the randomness factor to the transition progress was examined ${ }^{(1)}$. As a result, the process appeared first where the line spectral region increased gradually and the periodical fluctuation became dominant. The randomness factor decreased there. Thereafter, the line spectrum changed to the continuous spectrum, that is, the periodic fluctuations of the velocity changed to irregular fluctuations. The randomness factor increased

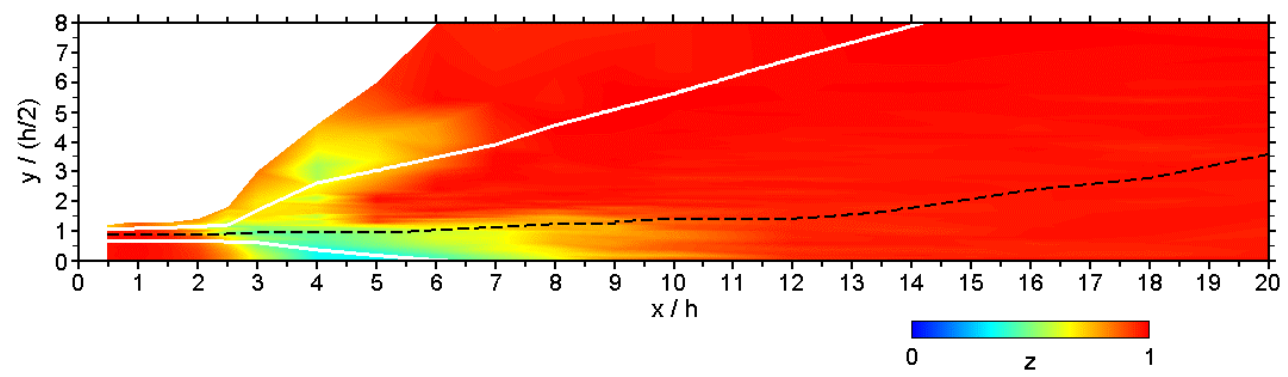

Fig. 6 Isocontour map of randomness factor. 
monotonically there. Thus, there is a problem that the randomness factor takes the same value in the two locations where the flow conditions are different.

In Fig. 6, the contour map of the randomness factor, $z$, in the $x-y$ plane is shown. The randomness factor was also shown in the range of $x / h \leqq 14$ in the previous report ${ }^{(1)}$, but is shown here in $x / h \leqq 20$. As a guide of the region where the mean velocity gradient $-\partial U / \partial$ $y$ exits, positions where $U / U_{m}$ equals 0.99 near the centerline $(y /(h / 2)=0)$ and 0.05 in the outer (large $y$ ) region are drawn as white solid lines, where $U_{m}$ is the velocity on the local centerline. The black dashed line is the $y /(h / 2)$ position where the rms value of the fluctuating velocity, $u$, becomes maximum respective of $x$. As can be seen from Fig. 6 , after it once decreased in the region of $3<x / h<5$, the randomness factor increased farther downstream, that is, it does not vary monotonically.

\subsection{Approximated Kolmogorov Complexity}

With the complexity analysis, it is possible to determine the randomness of the flow regardless of the change in spectral distribution. The results are shown in this and subsequent sections. First, the approximated Kolmogorov complexity (AK) that directly presents the data compressibility is examined. Analyzed data are the numerical data obtained from the first 32768 of 262144 data from the output voltage of the hot-wire anemometer, i.e., the streamwise fluctuating velocity, $u$. As described in Section 5.2, at first the minimum values are subtracted, then normalized by the common constant so as not to exceed unity and to remove the decimal point and negative sign, and finally converted to 3-digit integer each (total 98304 integer).

In Figs. 7-9, the contour map, distributions in the $x$ - and $y$-directions of AK are shown, respectively. In Fig. 8, variations are shown on two generators; one is on the centerline and the other is on the maximum position of $u$ ' in the distribution in $y$-direction at the respective $x$-station. In Fig. 7(b), the region just behind the nozzle exit where AK is small is only expanded and the color is drawn between the minimum and maximum range. The fact that $\mathrm{AK}$ is small shows that during the compression process any regularity is detected in the data, then the randomness becomes small. In the area around the dashed black line, AK is large, i.e., of greater complexity. The condition is quantitatively shown where regularity

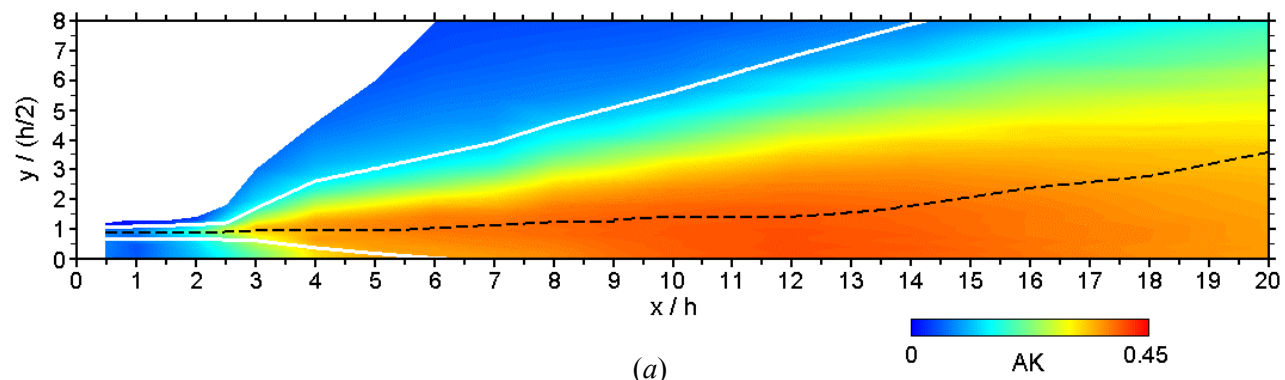

(a)

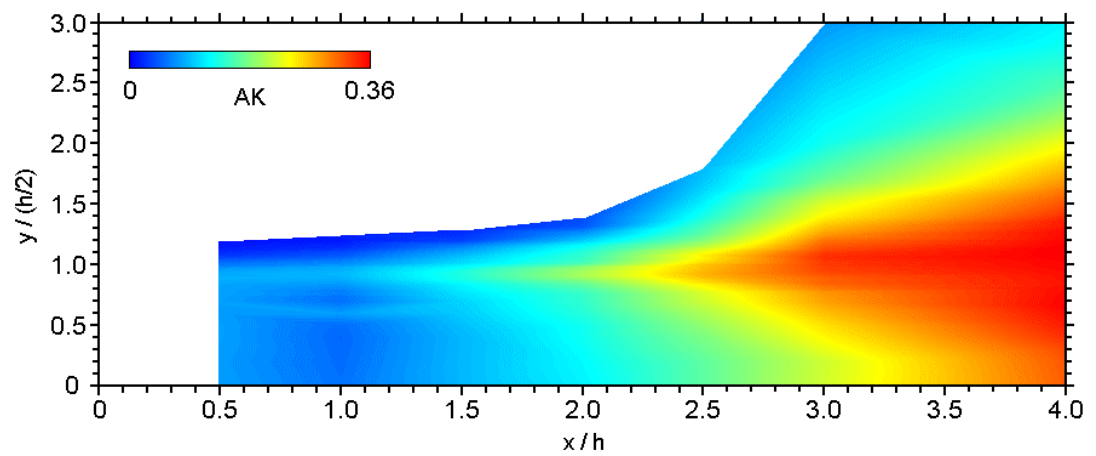

(b)

Fig. 7 Isocontour maps of approximated Kolmogorov complexity. 


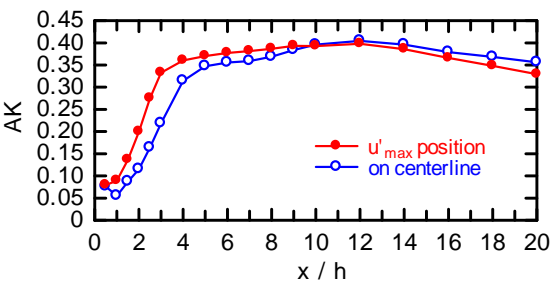

Fig. 8 Variations of approximated Kolmogorov complexity in streamwise direction.

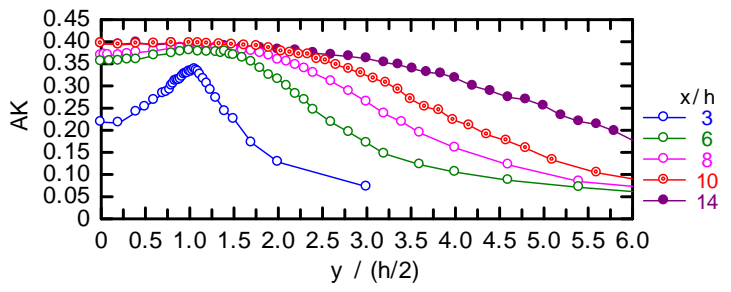

Fig. 9 Variations of approximated Kolmogorov complexity in $y$-direction.

remains in the flow before and immediately after the beginning of the transition, the regularity then disappears and the flow approaches random in accordance with the transition progress. Such upstream regularity is due to the smallness of the velocity fluctuation. Details of the transition progress including the fact that the flow just behind the nozzle exit is still laminar were given in the previous report ${ }^{(1)(49)}$.

In Fig. 8, after AK increased to $x / h=12$, it decreased slightly downstream. As shown in Fig. 6, the randomness factor reaches approximately unity at $x / h=16$, suggesting there is little contribution from a specific frequency to the fluctuating velocity. However, the randomness presented by the data compressibility rather decreases downstream. Since AK does not necessarily monotonically change, it is not possible to measure the turbulent transition by $\mathrm{AK}$ alone.

In Fig. 9, AK is large around the nozzle edge, $y /(h / 2)=1$, where the fluctuating velocity is also large, although it is small around the centerline at $x / h=3$ where the potential core still remains. Downstream, in $x / h \geqq 8, \mathrm{AK}$ is approximately constant over the $y$-region from the centerline to the position of maximum fluctuating velocity. It decreased monotonically with increasing $y$ thereafter. Hence, it can be seen that randomness decreases with the decrease in fluctuating velocity.

\subsection{NCD in Numerical Data of Fluctuating Velocity}

In this section, NCD was obtained. The numerical data are in the same form as in the previous section. In Figs. 10-12 of the contour map, distributions in the $x$ - and $y$-direction are shown, respectively. Values in the respective figures are $\operatorname{NCD}(X, Y)$ whose $Y$ data are taken at that position. The position of the reference data, $X$, is $x / h=0.5, y /(h / 2)=0$, as mentioned before. In order to investigate NCD just behind the nozzle in detail, Fig. 11 is drawn in a semi-logarithmic way. Since NCD at the reference position is 0.013 as shown in Table 1, the contour map of Fig. 10 is drawn with the exception of around the reference position. In the distribution on the centerline in Fig. 11, value rapidly increases from 0.013 in the reference position, $x / h=0.5$, to more than 0.9 in $x / h \geqq 1$.

In Fig. 10, NCD is greater in a region of great fluctuating velocity. Unlike AK and the randomness factor, in the streamwise variation in Fig. 11, after monotonically increasing, the value is also substantially constant, though scatters are rather large. Hence it does not take the same value in two positions, NCD is appropriate as a measure for the laminar-turbulent transition, though it reaches the maximum value at $x / h=4$ and 5 on the $u$ '-maximum position and on the centerline, respectively. In the downstream variation in the

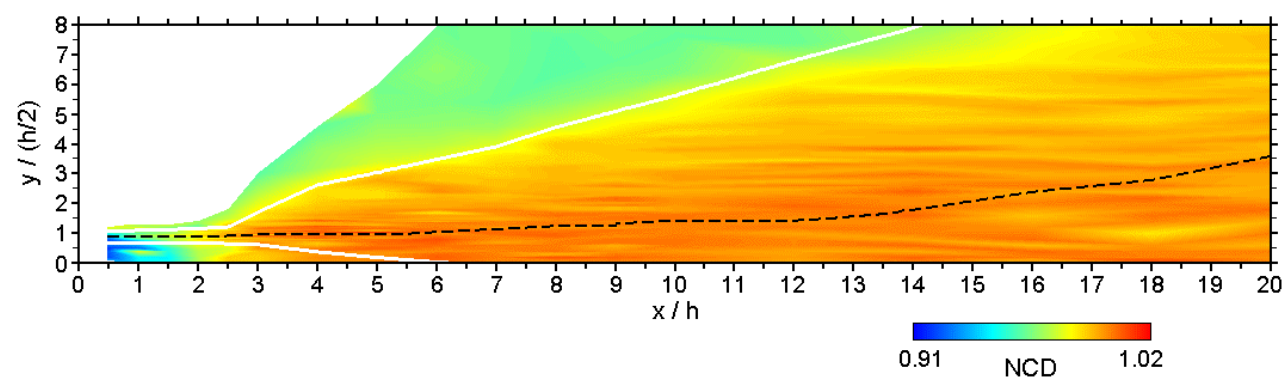

Fig. 10 Isocontour map of NCD between streamwise fluctuating velocities. 


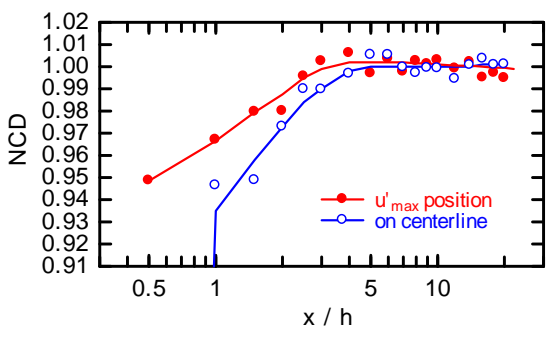

Fig. 11 Variations of NCD between streamwise fluctuating velocities in streamwise direction.

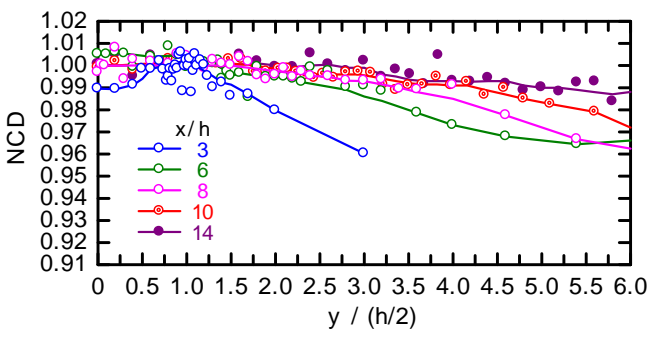

Fig. 12 Variations of NCD between streamwise fluctuating velocities in $y$-direction.

randomness factor the value greatly increased until $x / h=10$, then it gradually increased until $x / h=16$. In the downstream variation in AK (Fig. 8), the value increased until $x / h=$ 12. In contrast, the increase in NCD terminated at more upstream. In the distribution in the $y$-direction (Fig. 12), NCD varies similarly to AK in Fig. 9, though the scatter of the data is larger.

\subsection{NCD in Image Data of Fluctuating Velocity}

In this section, NCD of image data was obtained. As mentioned in Section 5.2, the image is displayed as a scatter plot of 262144 points of fluctuating velocities in the range of $-1 \leqq u / U_{0} \leqq 1$ and $-1 \leqq v / U_{0} \leqq 1$, where $U_{0}$ is the centerline velocity just after the nozzle exit, $x / h=0.5$. In Fig. 13, the scatter plots at the reference position, $y$-position of maximum $u$ ' at $x / h=3, y /(h / 2)=1$, and maximum $u$ ' position in the whole measurement range, $x / h=8, y /(h / 2)=1.3$ are shown as examples. As the reference position in Fig. 13(a) is in laminar flow on the centerline just behind the nozzle, many points are scattered only within a very narrow range around $u / U_{0}=0$ and $v / U_{0}=0$. On the other hand, as the transition progresses, the points begin to spread in a wide range with the increase of the fluctuating velocity. In Fig. 13(c), the spread is fairly wide and the points rarely overlap.

Excluding all other than the scattered points, such as borders of four sides, score lines, and axis labels and numbers in Fig. 13, the image of each 400 pixels in width and height was used to calculate NCD.

In the same manner as Section 5.3, the change of NCD with the number of the scattered points was checked. In the laminar region, NCD was almost constant irrespective of the number, whereas in the turbulent region NCD initially increased with the numbers, but soon reached saturation as in Fig. 3. In a laminar flow, as in Fig. 13(a), the points are scattered within a narrow range, and the distribution pattern is almost unchanged even if the number increases. On the other hand, in a turbulent flow, as points are distributed to a wide range as in Fig. 13(c), if the number is small the points are sparse and widespread, but as the number increases the points are closely deposited on the mass. Hence, 262144 points, with which NCD was well saturated in the turbulent flow, were scattered in this study.

In Figs. 14-16 of the contour map, distributions in the $x$ - and $y$-direction are shown, respectively. Similar to the numerical data in Fig. 10, NCD in the contour map, Fig. 14, is

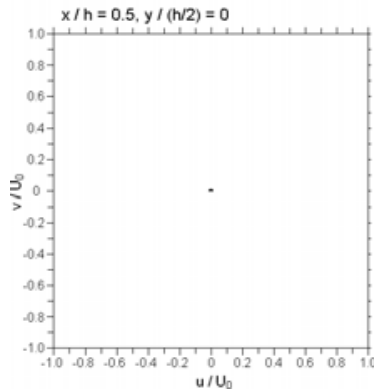

(a) Reference position

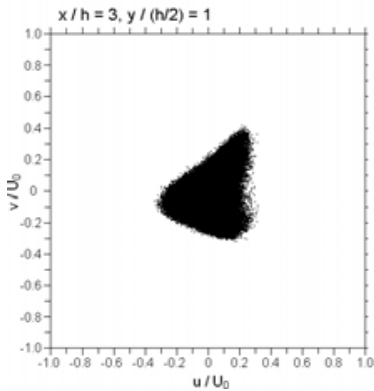

(b) $u^{\prime}{ }_{\text {max }}$ position at $x / h=3$

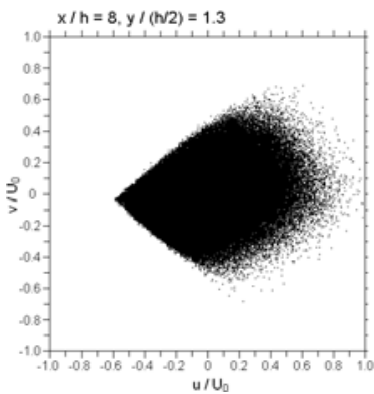

(c) $u^{\prime}{ }_{\max }$ position at $x / h=8$

Fig. 13 Scatter plots on $u-v$ plane. 


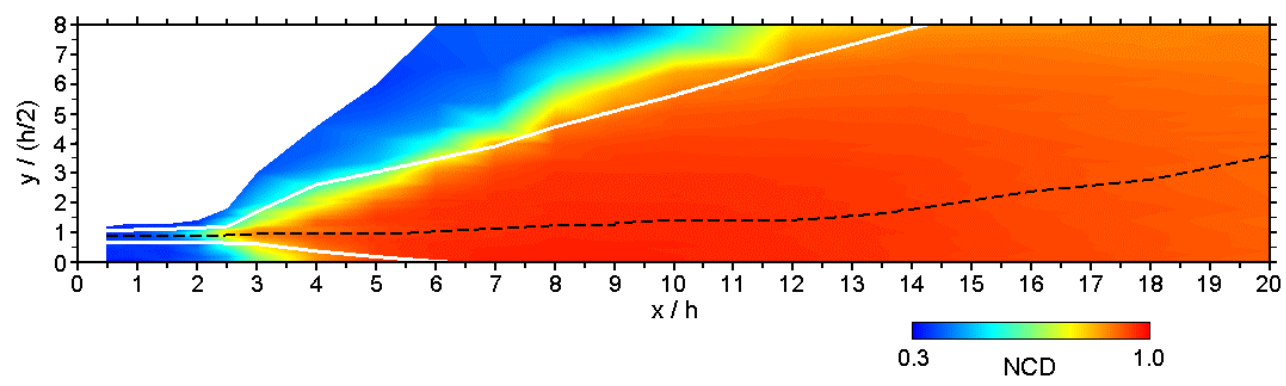

Fig. 14 Isocontour map of NCD between scatter plots.

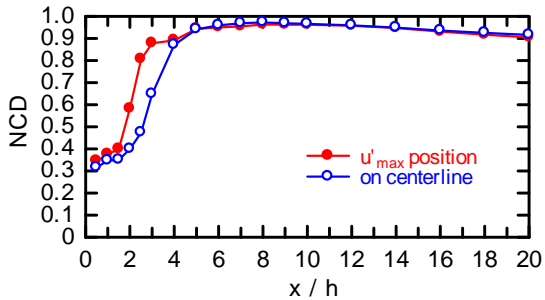

Fig. 15 Variations of NCD between scatter plots in streamwise direction.

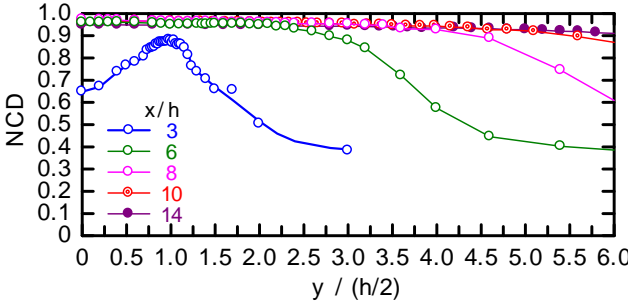

Fig. 16 Variations of NCD between scatter plots in $y$-direction.

greater in the greater fluctuation region. Contrary to the numerical data in Fig. 11, in the downstream distribution in Fig. 15, the value increased until $x / h \simeq 10$, then decreased downstream. Therefore, unlike NCD of the numerical data, questions remain as to the use of $\mathrm{NCD}$ of the image data as a measure for the laminar-turbulent transition progress. In the distribution in the $y$-direction in Fig. 16, the value is less dispersed than in the numerical data of Fig. 12, though similar variation is noted as in Fig. 9 and numerical NCD in Fig. 12.

\section{Conclusions}

Previous research on randomness and the definition of turbulence were reviewed. Then, Kolmogorov complexity and its approximation were explained as a measure for the representation of the randomness of finite discrete strings. In order to evaluate the randomness of the flow quantitatively, an application of finite discrete strings of digitally converted hot-wire output, and two-dimensional scatter plot images were proposed. In practice, the real numerical and image data in the laminar-turbulent transition process in the mixing layer formed downstream of the two-dimensional nozzle exit are compressed by the compression program, then the approximated Kolmogorov complexity and normalized compression distance were calculated. The following conclusions were obtained.

(1) For the present purposes, the $7 \mathrm{z}$ compression format is optimal among the range of the six formats examined.

(2) NCD for the numerical data of hot-wire anemometer output varies depending on the number of data. As the number increases it approaches zero between the same data and approaches unity between different data.

(3) AK can express randomness in turbulent flows and regularity in laminar flows quantitatively.

(4) NCD of numerical data from hot-wire anemometer output in the natural transition process of the mixing layer varies monotonically in the downstream direction. It can be a measure of the transition progress.

\section{References}

(1) Ichimiya, M., Kamada, S., Okajima, A., and Osaki, T., Effect of Local Periodic Disturbance on Mixing Layer Downstream of Two-Dimensional Jet (Spatial Structure and 
Quantitative Representation of Laminar-Turbulent Transition Process), Journal of Fluid Science and Technology, Vol. 8, No. 1 (2013), pp. 90-105.

(2) Sato, H., Laminar-Turbulent Transition in Free Shear Flow, in Tani, I. ed., Progress in Fluid Mechanics -Turbulent Flow- (1980), pp. 75, Maruzen (in Japanese).

(3) Kolmogorov, A. N., Combinatorial Foundations of Information Theory and the Calculus of Probabilities, Russian Mathematical Surveys, Vol. 38, No. 4 (1983), pp. 29-40.

(4) Tsuji, Y., and Nakamura, I., The Fractal Aspect of an Isovelocity Set and Its Relationship to Bursting Phenomena in the Turbulent Boundary Layer, Physics of Fluids, Vo. 6, No. 10 (1994), pp. 3429-3441.

(5) Ichimiya, M., Structure of a Turbulence Wedge Developed from a Single Roughness Element on a Flat Plate, in Rodi, W., and Martelli, F., ed., Engineering Turbulence Modelling and Experiments 2 (1993), pp.613-622, Elsevier.

(6) Hof, B., de Lozar, A., Avila, M., Tu, X., and Schneider, T. M., Eliminating Turbulence in Spatially Intermittent Flows, Science, Vol. 327 (2010), pp. 1491-1494.

(7) Ichimiya, M., Fujimura, H., and Tamatani, J., Laminar-Turbulent Transition of an Inlet Boundary Layer in a Circular Pipe Induced by Periodic Ejection (Condition for Generating an Isolated Turbulent Patch), Journal of Fluid Science and Technology, Vol. 6, No. 6 (2011), pp. 902-915.

(8) Ichimiya, M., Matsudaira, H., Fujimura, H., and Ohno, H., Laminar-Turbulent Transition of an Inlet Boundary Layer in a Circular Pipe Induced by Periodic Injection (Shape of Isolated Turbulent Patches and Their Growth), Transactions of the Japan Society of Mechanical Engineers, Series B, Vol. 79, No. 797 (2013), pp. 22-37 (in Japanese).

(9) Ichimiya, M., Matsudaira, H., and Fujimura, H., Laminar-Turbulent Transition of an Inlet Boundary Layer in a Circular Pipe Induced by Periodic Injection (Turbulence within Isolated Turbulent Patches and Its Growth Mechanism), Transactions of the Japan Society of Mechanical Engineers, Series B, Vol. 79, No. 801 (2013), pp. 863-878 (in Japanese).

(10) Prandtl, L., Führer durch die Strömungslehre 6.Auflage (1965), Friedr. Vieweg \& Sohn, (translated in Japanese, Shirakura, M., Tachibana, F., Akiyama, M., Shioya, S., Sanogawa, Y., and Fukui, S., Fluid Mechanics Vol. 1 (1972), pp. 128, Corona-sha).

(11) Tani, I., Fluid Mechanics 3rd. ed. (1967), pp. 128, Iwanami Shoten (in Japanese).

(12) Reynolds, O., An Experimental Investigation of the Circumstances Which Determine Whether the Motion of Water Shall be Direct or Sinuous, and of the Law of Resistance in Parallel Channels, Philosophical Transactions of the Royal Society of London, Vol. 174, (1883), pp. 935-982.

(13) Schlichting, H. and Gersten, K., Boundary-Layer Theory, 8th Revised and Enlarged ed. (2000), p. 13, Springer.

(14) Batchelor, G. K., The Theory of Homogeneous Turbulence (1956), Cambridge at the University Press.

(15) Frish, U., Turbulence, The Legacy of A. N. Kolmogorov (1995), Cambridge University Press.

(16) Hinze, J. O., Turbulence, 2nd ed. (1975), p. 2, McGraw-Hill.

(17) Townsend, A. A., The Structure of Turbulent Shear Flow, 2nd ed.(1976), p. 1, Cambridge University Press.

(18) Tennekes, H. and Lumley, J. L., A First Course in Turbulence (1972), pp. 1-4, The MIT Press.

(19) Nakamura, I., Turbulence Phenomena (1992), p. 15, Asakura Shoten (in Japanese).

(20) Nakamura, I., Miyata, M., Kushida, T., and Kagiya, K., An Experiment on the Intermittent Region of a Corner Turbulent Boundary Layer, Transactions of the Japan Society of Mechanical Engineers, Series B, Vol. 52, No. 479 (1986), pp. 2553-2559 (in Japanese).

(21) Lorenz, E. N., Deterministic Nonperiodic Flow, Journal of the Atmospheric Sciences, Vol. 20, Issue 2 (1963), pp. 130-141.

(22) Ruelle, D., Chance and Chaos (1991), Princeton University Press, (translated in Japanese, Aoki, K., (1993), Iwanami Shoten).

(23) Pope, S. B., Turbulent Flows (2000), p. 34, Cambridge University Press.

(24) Laplace, P.-S., Essai Philosophique sur les Probabilités (1814), (translated in Japanese, Uchii, S., Philosophical Essay on Probabilities (1997), Iwanami Shoten).

(25) Poincaré, H., Science et Méthode (1908), Ernest Flammarion, (translated in Japanese, Yoshida, Y., Science and Method (1950), Sogensha).

(26) Kolmogorov, A. N., Основные понятия теории вероятностей (1974) Hayka, (translated 
in Japanese, Nemoto, S., Basic Concepts of Probability Theory (1988), Tokyo Tosho).

(27) Li, M., and Vitányi, P. M. B., Kolmogorov Complexity and its Applications, in van Leeuwen, J. ed., Handbook of Theoretical Computer Science Vol. A, Algorithms and Complexity (1990) Elsevier, (translated in Japanese, Watanabe, O., Kolmogorov Complexity and its Applications, in Hirose, K., Nozaki, A., and Kobayashi, K. ed., Handbook of Theoretical Computer Science I(1994), Maruzen).

(28) Li, M., and Vitányi, P. M. B., An Introduction to Kolmogorov Complexity and its Applications, 2nd ed. (1997), Springer.

(29) Martin-Löf, P., The Definition of Random Sequences, Information and Control, Vol. 9, Issue 6 (1966), pp. 602-619.

(30) Watanabe, O., Defining Randomness, Mathematical Sciences, No. 519 (2006), pp. 10-15 (in Japanese).

(31) Watanabe, O., Randomness viewed from Computation Theory, Statistical Mathematics, Vol. 54, No. 2 (2006), pp. 511-523 (in Japanese).

(32) Nakamura, I., and Sakai, Y., Turbulent Diffusion, in The Japan Society of Fluid Mechanics ed., Handbook of Fluid Mechanics 2nd. ed. (1998), pp. 370-375, Maruzen (in Japanese).

(33) Sakai, Y., Nakamura, I., Tsunoda, H., and Hanabusa, K., Diffusion in Turbulent Pipe Flow Using the Stochastic Model, JSME International Journal, Series B, Vol. 39, No. 4 (1996), pp. 667-675.

(34) Tsuji, Y., Nakamura, I., and Kawachi, N., Simulation of Turbulent Velocity with Fractional Brownian Motion, Transactions of the Japan Society of Mechanical Engineers, Series B, Vol. 61, No. 588 (1995), pp. 2861-2868 (in Japanese).

(35) Gurzadyan, V. G., Kolmogorov Complexity as a Descriptor of Cosmic Microwave Background Maps, Europhysics Letters, Vol. 46, No. 1 (1999), pp. 114-117.

(36) Gell-Mann, M., and Lloyd, S., Information Measures, Effective Complexity, and Total Information, Complexity, Vol. 2, No. 1 (1996), pp. 44-52.

(37) Gell-Mann, M., and Lloyd, S., Effective Complexity, in Gell-Mann, M., and Tsallis, C., ed., Nonextensive Entropy-Interdisciplinary Applications (2003), pp. 387-398, Oxford University Press.

(38) Takeda, G., Complexity and Science, Journal of the Japan Association for Philosophy of Science, Vol. 26, No. 1 (1998), pp. 1-7 (in Japanese).

(39) Kreinovich, V., and Kunin, I. A., Kolmogorov Complexity and Chaotic Phenomena, International Journal of Engineering Science, Vol.41, Issues 3-5 (2003), pp. 483-493.

(40) Badii, R., and Politi, A., Complexity (1997), Cambridge University Press, (translated in Japanese, Aizawa, Y., Mathematical Sciences of Complexity (2001), pp. 14-21, Sangyo Tosho).

(41) Rissanen, J. J, Fisher Information and Stochastic Complexity, IEEE Transactions on Information Theory, Vol. 42, No. 1 (1996), pp. 40-47.

(42) Vitányi, P., (translated in Japanese, Watanabe, O., Universal Similarity Measurement Based on Complexity, Mathematical Sciences, No. 519 (2006), pp. 54-59).

(43) Nakamura, I., and Watanabe, T., Qualitative Physics, in The Japan Society of Mechanical Engineers ed., Fluid Informatics (2010), pp. 58-69, Gihodo (in Japanese).

(44) Li, M., Chen, X., Li, X., Ma, B., and Vitányi, P. M. B., The Similarity Metric, IEEE Transactions on Information Theory, Vol. 50, No. 12 (2004), pp. 3250-3264.

(45) Bannai, K., Narisawa, K., Brodkorb, F., and Shinohara A., Similarity Measure using Lossy Compression and its Application to Image Retrieval, Proceedings of Forum on Information Technology 2010, Vol. 1 (2010), pp. 215-220 (in Japanese).

(46) Fujiwara, Y., Gotoh, T., and Iguchi H., Product/Service Value Validation based on Kolmogorov Complexity, Proceedings of Forum on Information Technology 2009, Vol. 2 (2009), pp. $55-62$ (in Japanese).

(47) Cilibrasi, R. L., and Vitányi, P. M. B., The Google Similarity Distance, IEEE Transactions on Knowledge and Data Engineering, Vol. 19, No. 3 (2007), pp. 370-383.

(48) Matsumoto, M., and Nishimura, T., Mersenne Twister: A 623-Dimensionally Equidistributed Uniform Pseudorandom Number Generator, ACM Transactions on Modeling and Computer Simulations, Vol. 8, No. 1 (1998), pp. 3-30.

(49) Ichimiya, M., Kato, T., and Morimoto, T., Effect of Local Periodic Disturbance on Mixing Layer at Exit of Two-Dimensional Jet, Journal of Fluid Science and Technology, Vol. 6, No. 6 (2011), pp. 887-901. 